\title{
The VVDS-VLA deep field
}

\section{Optical and near infrared identifications of VLA $S_{1.4} \mathrm{GHz}>80 \mu \mathrm{Jy}$ sources in the VIMOS VLT deep survey VVDS-02h field}

\author{
P. Ciliegi ${ }^{1}$, G. Zamorani ${ }^{1}$, M. Bondi ${ }^{2}$, L. Pozzetti ${ }^{1}$, M. Bolzonella ${ }^{3}$, L. Gregorini ${ }^{2,4}$, B. Garilli ${ }^{5}$, A. Iovino ${ }^{6}$, \\ H. J. McCracken ${ }^{7,8}$, Y. Mellier ${ }^{7,8}$, M. Radovich ${ }^{9}$, H. R. de Ruiter ${ }^{1}$, P. Parma ${ }^{2}$, D. Bottini ${ }^{5}$, V. Le Brun ${ }^{10}$, \\ O. Le Fèvre ${ }^{10}$, D. Maccagni ${ }^{5}$, J. P. Picat ${ }^{12}$, R. Scaramella ${ }^{11}$, M. Scodeggio ${ }^{5}$, L. Tresse ${ }^{10}$, G. Vettolani $^{2}$, \\ A. Zanichelli ${ }^{2}$, C. Adami ${ }^{10}$, M. Arnaboldi ${ }^{9}$, S. Arnouts ${ }^{10}$, S. Bardelli ${ }^{1}$, A. Cappi ${ }^{1}$, S. Charlot ${ }^{8}$, T. Contini ${ }^{12}$, \\ S. Foucaud ${ }^{5}$, P. Franzetti ${ }^{5}$, L. Guzzo ${ }^{6}$, O. Ilbert ${ }^{10}$, B. Marano ${ }^{3}$, C. Marinoni ${ }^{10}$, G. Mathez ${ }^{12}$, A. Mazure ${ }^{10}$, \\ B. Meneux ${ }^{10}$, R. Merighi ${ }^{1}$, P. Merluzzi ${ }^{9}$, S. Paltani ${ }^{10}$, A. Pollo ${ }^{6}$, E. Zucca ${ }^{1}$, A. Bongiorno ${ }^{3}$, G. Busarello9, \\ I. Gavignaud ${ }^{12}$, R. Pellò̀ ${ }^{12}$, V. Ripepi ${ }^{9}$, and D. Rizzo ${ }^{12}$
}

1 INAF - Osservatorio Astronomico di Bologna, via Ranzani 1, 40127 Bologna, Italy e-mail: paolo.ciliegi@bo.astro.it

2 IRA - INAF, via Gobetti 101, 40129 Bologna, Italy

3 Università di Bologna, Dipartimento di Astronomia, via Ranzani 1, 40127 Bologna, Italy

4 Università di Bologna, Dipartimento di Fisica, via Irnerio 46, 40126 Bologna, Italy

5 IASF-INAF, via Bassini 15, 20133 Milano, Italy

6 INAF - Osservatorio Astronomico di Brera, via Brera 28, Milan Italy

7 Institut d'Astrophysique de Paris, UMR 7095, 98 bis Bd Arago, 75014 Paris, France

8 Observatoire de Paris, LERMA, 61 avenue de l'Observatoire, 75014 Paris, France

9 INAF - Osservatorio Astronomico di Capodimonte, via Moiariello 16, 80131 Napoli, Italy

${ }^{10}$ Laboratoire d'Astropysique de Marseille, UMR 6110 CNRS - Université de Provence, Traverse du Siphon-les Trois Lucs, 13012 Marseille, France

11 INAF - Osservatorio Astronomico di Roma, Roma, Italy

12 Laboratoire d'Astrophysique de l'Observatoire Midi-Pyrénées (UMR 5572), 14 avenue E. Belin, 31400 Toulouse, France

Received 19 October 2004 / Accepted 9 June 2005

\section{ABSTRACT}

In this paper we present the optical and near-infrared identifications of the 1054 radio sources detected in the $20 \mathrm{~cm}$ deep radio survey down to a $5 \sigma$ flux limit of $\sim 80 \mu \mathrm{Jy}$ obtained with the VLA in the VIMOS VLT Deep Survey VVDS-02h deep field. Using $U, B, V, R, I$ and $K$ data, with limiting magnitudes of $U_{A B} \sim 25.4, B_{A B} \sim 26.5, V_{A B} \sim 26.2, R_{A B} \sim 25.9 I_{A B} \sim 25.0, J_{A B} \sim 24.2, K_{A B} \sim 23.9$ (50\% completeness) we identified 718 radio sources ( $\sim 74 \%$ of the whole sample).

The photometric redshift analysis shows that, in each magnitude bin, the radio sample has a higher median photometric redshift than the whole optical sample, while the median $(V-I)_{A B}$ color of the radio sources is redder than the median color of the whole optical sample. These results suggest that radio detection is preferentially selecting galaxies with higher intrinsic optical luminosity.

From the analysis of the optical properties of the radio sources as function of the radio flux, we found that while about $35 \%$ of the radio sources are optically unidentified in the higher radio flux bin $(S>1.0 \mathrm{mJy})$, the percentage of unidentified sources decreases to about $25 \%$ in the faintest bins $\left(S<0.5 \mathrm{mJy}\right.$ ). The median $I_{A B}$ magnitude for the total sample of radio sources, i.e. including also the unidentified ones, is brighter in the faintest radio bins than in the bin with higher radio flux. This suggests that most of the faintest radio sources are likely to be associated to relatively lower radio luminosity objects at relatively modest redshift, rather than radio-powerful, AGN type objects at high redshift. Using a classification in early-type and late-type galaxies based on the $(B-I)_{A B}$ color and the photometric redshift, we found that the majority of the radio sources below $\sim 0.15 \mathrm{mJy}$ are indeed late-type star forming galaxies. Finally, the radio sources without optical counterpart in our deep imaging have a median radio flux of $0.15 \mathrm{mJy}$, equal to that of identified sources. Given the very faint optical limits, these unidentified radio sources probably contain a significant fraction of obscured and/or high redshift galaxies.

Key words. cosmology: observations - galaxies: general - galaxies: starburst - radio continuum: galaxies 


\section{Introduction}

Deep 1.4-GHz counts show an upturn below a few millijansky (mJy), corresponding to a rapid increase in the number of faint sources. Photometric and spectroscopic studies suggest that the faint excess at $1.4 \mathrm{GHz}$ is composed predominantly of star-forming galaxies, with a contribution also from low-power AGNs and early type galaxies. However, despite many dedicated efforts (see for example Benn et al. 1993; Hammer et al. 1995; Gruppioni et al. 1999; Richards et al. 1999; Prandoni et al. 2001) the relative fraction of the various populations responsible of the sub-mJy radio counts (AGN, starburst, late and early type galaxies), are not well established yet. In fact, the photometric and spectroscopic work needed in the optical identification process is very demanding in terms of telescope time, since a significant fraction of faint radio sources have also very faint optical counterparts. It is therefore clear that in order to investigate the nature and evolution of the sub-mJy population it is absolutely necessary to couple deep radio and optical (both imaging and spectroscopic) observations over a reasonably large area of the sky.

The VVDS-VLA Deep survey is one of the best available surveys to investigate the nature of the sub-mJy population. A deep radio survey has been obtained in $1 \mathrm{deg}^{2}$ with the VLA down to a $5 \sigma$ flux limit of $\sim 80 \mu \mathrm{Jy}$ (Bondi et al. 2003). A deep multi-color $B V R I$ photometric survey of the whole area is available (Le Fèvre et al. 2004a; McCracken et al. 2003), together with $U$ band (Radovich et al. 2004) and $J$ and $K$ band (Iovino et al. 2005) data. Furthermore, a deep spectroscopic survey is being performed with the VIMOS spectrograph at the VLT (Le Fèvre et al. 2004b).

In this paper we present the optical identification of the VIMOS radio sources obtained with the photometric data in the $U, B, V, R, I$ and $K$ bands. In Sect. 2 we give a description of the available radio, optical and near infrared data, while the description of the technique used for the optical identification of the radio sources is presented in Sect. 3. Finally, Sect. 4 is a discussion of our results, while Sect. 5 summarizes our conclusion.

\section{The available data}

\subsection{Radio data}

The radio data were obtained with the Very Large Array (VLA) in B configuration. A 1 square degree mosaic map with an approximately uniform noise of $17.5 \mu \mathrm{Jy}(1 \sigma)$ and with a $6 \times 6$ arcsec $F W H M$ Gaussian resolution beam has been obtained. This map (centered at $\mathrm{RA}(\mathrm{J} 2000)=02: 26: 00$ $\operatorname{Dec}(\mathrm{J} 2000)=-04: 30: 00)$ has been used to extract a complete catalogue of 1054 radio sources, 19 of which are considered as multiple, i.e. fitted with at least two separate component. A detailed description of the radio observations, data reduction, sources extraction and radio source counts is reported in Bondi et al. (2003).

\subsection{The optical and near infrared data in the $U, B, V, R, I$ and $K$ bands}

Almost the whole square degree of the VVDS-VLA field has been observed in the $B, V, R$ and $I$ bands with the CFH12K wide-field mosaic camera during the CFH12K-VIMOS deep imaging survey (Le Fèvre et al. 2004a). These observations reach limiting magnitudes (50\% completeness for point sources) of $B_{A B} \sim 26.5, V_{A B} \sim 26.2, R_{A B} \sim 25.9$ and $I_{A B} \sim$ 25.0. A detailed description of these optical data is given in McCracken et al. (2003). Moreover, a $U$ band survey has been carried out with the wide field imaging (WFI) mosaic camera mounted on the ESO MPI 2.2 m telescope at La Silla, Chile. The total effective area covered so far by the $U$ band survey is $\sim 0.71 \mathrm{deg}^{2}$. The limiting magnitude of the catalogue obtained with these observations is 25.4 in $U_{A B}$ (50\% completeness). A detailed description of the VIMOS $U$ band imaging survey is reported in Radovich et al. (2004). Finally, a fraction of the VVDS-VLA field has been observed in the $J$ and $K$ bands with the SOFI instrument mounted on the ESO NTT telescope. The total area covered by the $J$ and $K$ bands survey is $\sim 165 \mathrm{arcmin}^{2}$, down to limiting magnitudes of $J_{A B} \sim 24.2$ and $K_{A B} \sim 23.9$ (50\% completeness for point sources). A detailed description of the VIMOS $J$ and $K$ band imaging survey is reported in Iovino et al. (2005).

In order to obtain a $B_{A B}, V_{A B}, R_{A B}, I_{A B}$ catalogue, all the images were combined to obtain a unique detection image using the $\chi^{2}$ technique (Szalay et al. 1999). Subsequently, the photometry in each bandpass has been performed at the position defined in the $\chi^{2}$ image. The primary advantage in using this technique is to simplify the generation of multi-band catalogues and to reduce the number of spurious detections. For a detailed description of the $B_{A B}, V_{A B}, R_{A B}, I_{A B}$ catalogue see McCracken et al. (2003). Subsequently, the $\chi^{2}$ image has been updated with the $U_{A B}, J_{A B}$ and $K_{A B}$ data (in the areas covered in these bands) using the same technique described by McCracken et al. (2003).

\section{Optical identification}

\subsection{Radio-optical off-set}

The relative off-set between the radio and optical catalogue has been estimated using the radio position of the 160 unresolved radio sources with a peak flux density greater than $0.17 \mathrm{mJy}$ (i.e. detected with peak flux $\sim 10 \sigma$ ) identified with point like optical counterparts. Their $\Delta \mathrm{RA}$ and $\Delta \mathrm{Dec}$ offset are shown in Fig. 1. The mean offsets, with their standard errors $(\sigma / \sqrt{N})$, are $\langle\Delta \mathrm{RA}\rangle=+0.13 \pm 0.03^{\prime \prime}$ and $\langle\Delta \mathrm{Dec}\rangle=-0.32 \pm 0.03^{\prime \prime}$. These offsets should be removed (subtracting $0.13^{\prime \prime}$ in RA and adding $0.32^{\prime \prime}$ in Dec at the radio position) to obtain the radio position in the same reference frame as the optical CCD.

\subsection{Optical identification of the radio sources}

Of the 1054 radio sources in the complete radio sample, 57 are outside the presently available catalogues based on our CCD data. The optical identification of these 57 sources with the 


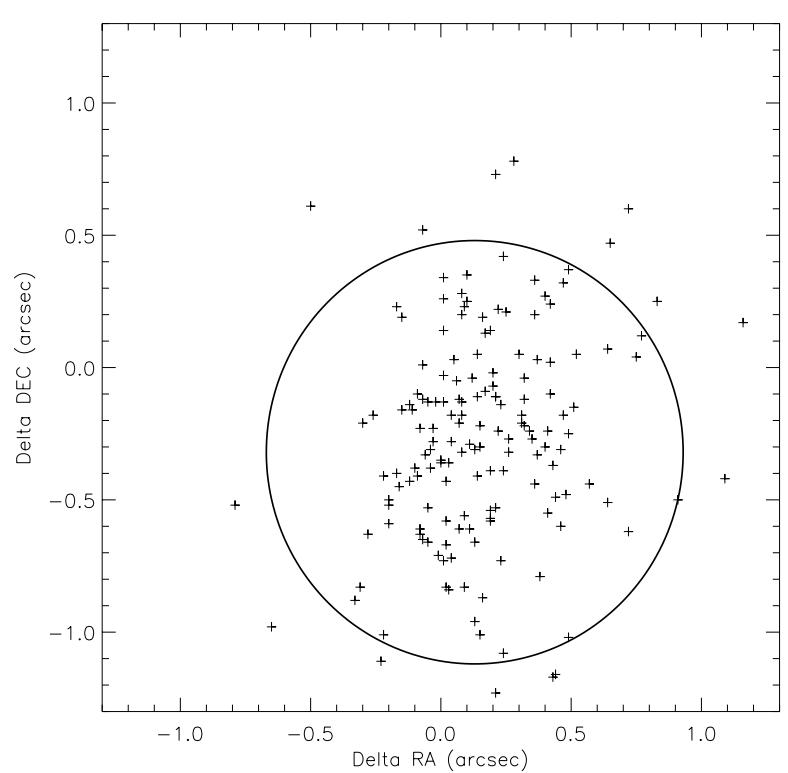

Fig. 1. Position offsets for strong $(>10 \sigma)$ radio point-like sources identified with a point-like optical counterparts. The circle of radius 0.8 arcsec (with the mean offset removed) encloses $90 \%$ of the sources.

available public catalogues is described in the next section. Moreover, 24 radio sources are within the masked regions in the $U B V R I K$ catalogue, since they are spatially coincident with bright stars or their diffraction spikes. The total number of radio sources for which optical data are available in the VIMOS CCD catalogue is therefore 973.

For the optical identification of these 973 radio sources, we used the likelihood ratio technique, first used in this context by Richter (1975) and in modified form by de Ruiter et al. (1977), Prestage \& Peacock (1983), Sutherland \& Saunders (1992) and Ciliegi et al. (2003). The mean off-set between the radio and optical positions estimated in the previous section has been removed from the radio positions to compute the positional offset.

The likelihood ratio $L R$ is the ratio between the probability that a given source at the observed position and with the measured magnitude is the true optical counterpart, and the probability that the same source is a chance background object:

$L R=\frac{q(m) f(r)}{n(m)}$

where $q(m)$ is the expected distribution as a function of magnitude of the optical counterparts, $f(r)$ is the probability distribution function of the positional errors, while $n(m)$ is the surface density of background objects with magnitude $m$ (see Ciliegi et al. 2003, for a detailed discussion on the procedure to calculate $q(m), f(r)$ and $n(m))$. For each source we adopted an elliptical Gaussian distribution for the positional errors with the errors in RA and Dec on the radio position reported in the radio catalogue (Bondi et al. 2003) and assuming a value of 0.3 arcsec as optical position uncertainty (McCracken et al. 2003).

The presence or absence of more than one optical candidates for the same radio source provides additional information to that contained in $L R$. The reliability $\operatorname{Rel}_{j}$ for object $j$ being the correct identification is (Sutherland \& Saunders 1992):

$\operatorname{Rel}_{j}=\frac{(L R)_{j}}{\Sigma_{i}(L R)_{i}+(1-Q)}$

where the sum is over the set of all candidates for this particular source and $Q$ is the probability that the optical counterpart of the source is brighter than the magnitude limit of the optical catalogue $\left(Q=\int^{m_{\lim }} q(m) \mathrm{d} m\right)$.

The adopted value for $Q$ is 0.65 . This value has been estimated by the comparison between the expected number of identifications (658) derived from the integral of the $q(m)$ distribution and the number of the radio sources (973) that we used in the Likelihood Ratio technique (see Ciliegi et al. 2003, for more details). However, to check how this assumption could affect our results, we repeated the likelihood ratio analysis using different values of $Q$ in the range $0.6-0.8$. No substantial difference in the final number of identifications and in the associated reliability has been found.

Once $q(m), f(r)$ and $n(m)$ were obtained, we computed the $L R$ value for all the optical sources within a distance of 5 arcsec from the radio position. Having determined the $L R$ for all the optical candidates, one has to choose the best threshold value for $L R\left(L_{\mathrm{th}}\right)$ to discriminate between spurious and real identifications. As $L R$ threshold we adopted $L_{\mathrm{th}}=0.35$. With this value, according to Eq. (2) and considering that our estimate for $Q$ is 0.65 , all the optical counterparts of radio sources with only one identification (the majority in our sample) and $L R>L R_{\text {th }}$ have a reliability greater than 0.5 . This choice also approximately maximizes the sum of sample reliability and completeness. With this threshold value we find 718 radio sources with a likely identification, 14 of which have two optical candidates with $L_{\mathrm{th}}>0.35$ for a total of 732 optical candidates with $L_{\mathrm{th}}>0.35$. The maximum distance between the radio and optical position is 2.98 arcsec. The number of expected real identifications (obtained by summing the reliability of all the objects with $L_{\text {th }}>0.35$ ) is about 683 , i.e. we expect that about 35 of the 718 proposed radio-optical associations may be spurious positional coincidences. In the 14 cases in which more than one optical object with $L R>L R_{\text {th }}$ has been found associated to the same radio source, we assumed the object with the highest Likelihood Ratio value as the counterpart of this radio source. Among the 255 unidentified radio sources, 17 are empty fields (i.e. they have no optical source within 5 arcsec from their position), while the other 238 sources have at least one optical source within 5 arcsec, but all with $L R<L R_{\text {th }}$.

\subsection{Optical identification of the radio sources outside the VIMOS CCD catalogue}

As explained above, 57 radio sources lie outside the currently available VIMOS CCD catalogue. For the optical identification of these sources we used the Guide Sky Catalogue 2.2 ${ }^{1}$, which provides a two bandpass $(J \sim B$ and $F \sim R$ ) catalogue with a magnitude limit of $J \sim 19.5 \mathrm{mag}$ and $F \sim 18.5 \mathrm{mag}$. Using a maximum distance of 3 arcsec between the radio and optical

\footnotetext{
${ }^{1}$ http://www-gsss.stsci.edu/gsc/gsc2/GSC2home.htm
} 
Table 1. Optical properties of the 7 radio sources outside the CCD area identified with the GSC2.2 catalogue.

\begin{tabular}{lcccccccc}
\hline \hline Name & $\begin{array}{c}S_{\mathrm{T}} \\
(\mathrm{mJy})\end{array}$ & $\begin{array}{c}\Delta \\
\left({ }^{\prime \prime}\right)\end{array}$ & $J_{\mathrm{GSC} 2}$ mag & $F_{\mathrm{GSC} 2}$ mag & $\mathrm{c}$ & $J_{2 \text { mass }}$ & $H_{2 \text { mass }}$ & $K_{2 \text { mass }}$ \\
\hline VIMOS1.4GHz_J022412-045954 & 0.212 & 2.71 & 14.420 .34 & 14.590 .23 & 3 & 16.820 .19 & 16.140 .26 & $>14.78$ \\
VIMOS1.4GHz_J022526-040116 & 0.709 & 0.21 & 14.150 .42 & 15.290 .29 & 3 & 15.260 .10 & 14.410 .12 & 13.950 .09 \\
VIMOS1.4GHz_J022549-040023 & 2.125 & 0.41 & 13.470 .42 & 14.380 .29 & 0 & 14.740 .08 & 14.080 .10 & 13.660 .08 \\
VIMOS1.4GHz_J022603-045902 & 0.682 & 1.49 & 14.010 .37 & 15.040 .25 & 3 & 15.360 .09 & 14.500 .10 & 14.220 .08 \\
VIMOS1.4GHz_J022604-045932 & 1.079 & 1.57 & 13.010 .37 & 14.020 .25 & 3 & 14.860 .08 & 13.990 .09 & 13.500 .07 \\
VIMOS1.4GHz_J022609-045804 & 1.792 & 1.28 & 11.790 .37 & 13.100 .25 & 3 & 14.140 .08 & 13.530 .09 & 12.980 .07 \\
VIMOS1.4GHz_J022628-040051 & 1.257 & 0.16 & 17.030 .42 & 18.730 .30 & 3 & 16.190 .08 & 15.330 .11 & 14.730 .11 \\
\hline
\end{tabular}

Table 2. Summary of the VVDS-VLA radio sample identification in optical and near infrared bands.

\begin{tabular}{|c|c|c|c|c|c|c|c|c|c|c|}
\hline \multicolumn{11}{|c|}{ Whole radio sample: 1054 sources } \\
\hline \multicolumn{11}{|c|}{ Bands } \\
\hline Number of sources: & $U$ & $B$ & $V$ & $R$ & $I$ & $J$ & $K$ & $J_{2 \text { mass }}$ & $H_{2 \text { mass }}$ & $K_{2 \text { mass }}$ \\
\hline Outside CCD area & 415 & 65 & 66 & 63 & 63 & 989 & 989 & 0 & 0 & 0 \\
\hline Within masked regions & 2 & 24 & 24 & 24 & 24 & 0 & 0 & 0 & 0 & 0 \\
\hline With good CCD data & 637 & 965 & 964 & 967 & 967 & 65 & 65 & 1054 & 1054 & 1054 \\
\hline With reliable identification & 371 & 687 & 703 & 714 & 707 & 42 & 43 & 105 & 104 & 93 \\
\hline With reliable identification with $I_{A B}<24.0$ & 361 & 643 & 660 & 668 & 670 & 40 & 40 & 105 & 104 & 93 \\
\hline
\end{tabular}

positions (2.98 arcsec is the maximum radio-optical offset for the radio sources identified with the likelihood ratio technique, see previous section), we identified 7 radio sources, only one of which has a radio-optical offset greater than 2 arcsec. The optical properties of these 7 radio sources are given in Table 1. For each source we report the name, the $20 \mathrm{~cm}$ total radio flux, the distance between the optical and radio position, the $J$ and $F$ magnitude with relative errors and the optical classification c as reported in the GSC2.2 catalogue ( 0 for stellar and 3 for non stellar). Given the small number of sources ( 7 in comparison to the 718 identified within the CCD area) and the difference in the magnitude systems ( $J$ and $F$ in the GSC2 catalogue vs. $B_{A B}$ and $R_{A B}$ in the CCD catalogue), these 7 sources have not been considered in the analysis and discussion reported in the next sections and are not included in the identification summary reported in Table 2.

\subsection{Near-infrared identification}

Only 65 of the 1054 radio sources lie within the $\sim 165 \operatorname{arcmin}^{2}$ covered by our deep $K$ band survey: 21 are unidentified both in the optical and $K$ bands, 1 has a reliable optical counterpart but is unidentified in the $K$ band, while 43 have a reliable counterpart both in the optical and $K$ bands. To fully explore the nearinfrared properties of our radio sample, we searched also the Two Micron All Sky Survey (2MASS ${ }^{2}$, Cutri+ 2003) database for near-IR ( $J, H$ and $K$ bands) counterparts of our 1054 radio sources. The $3 \sigma$ limits of the 2MASS survey are 17.1, 16.4 and 15.3 mag respectively in the three bandpasses $J, H$ and $K$. The image pixel scale of the 2MASS detectors is $2.0^{\prime \prime}$, and the positional uncertainties are $\leq 0.5^{\prime \prime}$. Using a maximum

\footnotetext{
${ }^{2}$ http://www.ipac.caltech.edu/2mass/
}

distance of $3^{\prime \prime}$ between the radio and 2MASS positions, we found 105 reliable matches, with 105 detections in $J, 104$ in $H$, and 93 in $K$, including the 7 sources outside the optical CCD area reported in Table 1. Owing to the relatively shallow flux limits of the 2MASS survey, the surface density of background sources is low enough that with a maximum off-set of $3^{\prime \prime}$ between the radio and 2MASS positions, chance associations with radio positions are very unlikely. All the 2MASS sources have $I_{A B} \leq 19.0$. The $J, H$ and $K$ magnitudes have been converted to the $A B$ system using $J_{A B}=J+0.90, H_{A B}=H+1.37$ and $K_{A B}=K+1.84$.

\subsection{Identification summary}

A summary of the optical identifications is reported in Table 2. For each band we report the number of sources that lie outside the available CCD area, the number of sources within the masked regions (i.e. sources coincident with bright stars or with their diffraction spikes for which a reliable identification is impossible), the number of sources for which reliable data are available (1054 - outside CCD - within masked regions) and the number of sources with a reliable identification. Finally in the last line we report the number of reliable identifications with an $I_{A B}$ magnitude brighter than 24 , i.e. corresponding to the sources that are potential targets for the VIMOS spectroscopic survey (Le Fèvre et al. 2004b). The 7 radio sources identified in the GSC 2.2 catalogue are not included in Table 2.

In Fig. 2 we report the radio peak flux distributions for the whole radio sample, for the 718 radio sources with a reliable optical identification and for the 105 radio sources with a near infrared counterpart in the 2MASS survey. As clearly shown in the figure, while the peak flux distribution of radio sources with a 2 MASS counterpart is strongly 
Table 3. Photometric data for the identified radio sources: a portion is shown here for guidance regarding its form and content. The whole catalogue is available at the web page http://virmos.bo.astro.it/radio/catalogue.html.

\begin{tabular}{|c|c|c|c|c|c|c|c|}
\hline Name & $U_{A B}$ & $B_{A B}$ & $V_{A B}$ & $R_{A B}$ & $I_{A B}$ & $J_{A B}$ & $K_{A B}$ \\
\hline VIRMOS1.4GHz_J022400-040527 & $>26.45$ & $>26.35$ & $25.52 \pm 0.34$ & $24.66 \pm 0.14$ & $23.98 \pm 0.19$ & - & - \\
\hline VIRMOS1.4GHz_J022400-043009 & $>26.45$ & $>26.75$ & $25.45 \pm 0.22$ & $24.85 \pm 0.19$ & $22.76 \pm 0.05$ & - & - \\
\hline VIRMOS1.4GHz_J022400-044950 & - & $20.56 \pm 0.01$ & $19.22 \pm 0.01$ & $18.74 \pm 0.01$ & $18.15 \pm 0.01$ & - & - \\
\hline VIRMOS1.4GHz_J022401-040734 & $25.61 \pm 0.17$ & $23.19 \pm 0.04$ & $21.71 \pm 0.3$ & $20.59 \pm 0.01$ & $19.69 \pm 0.01$ & - & - \\
\hline VIRMOS1.4GHz_J022401-042621 & $24.89 \pm 0.12$ & $23.94 \pm 0.06$ & $23.05 \pm 0.05$ & $21.94 \pm 0.02$ & $21.17 \pm 0.02$ & - & - \\
\hline VIRMOS1.4GHz_J022402-040705 & $24.39 \pm 0.11$ & $24.03 \pm 0.07$ & $23.18 \pm 0.06$ & $23.81 \pm 0.09$ & $23.04 \pm 0.10$ & - & - \\
\hline
\end{tabular}

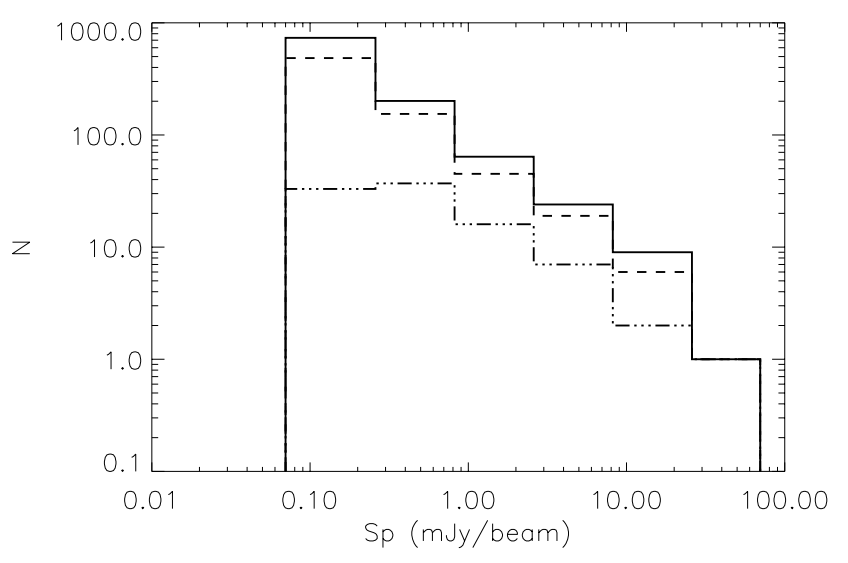

Fig. 2. Radio peak flux distribution for the whole radio sample (solid line), for the 718 radio sources with a reliable optical identification (dashed line) and for the 105 radio sources with a near infrared identification in the 2MASS survey (dot dot dot dashed line).

biased towards high radio flux (as expected due to the bright limits of the 2MASS survey), the flux peak distribution of the 718 radio sources with an optical identification does not show any statistically significant difference with respect to the distribution of the whole radio sample. The final catalogue of all 718 identified radio sources is available on the web at http://virmos . bo. astro.it/radio/catalogue.html. A sample of the catalogue is shown in Table 3.

\section{Discussion}

\subsection{Optical magnitude distributions}

In absence of spectroscopic data, the magnitude and color distributions of the optical counterparts can be used to derive some informations on the nature of faint radio sources. In Fig. 3 we show the magnitude distributions in the $U_{A B}, B_{A B}, R_{A B}$, and $I_{A B}$ bands of the optical counterparts of the radio sources as filled histograms. The empty histograms show the magnitude distributions of the whole optical data set.

This figure clearly shows that the magnitude distributions of the optical counterparts of the radio sources are significantly flatter than those of the global optical catalogues, reaching a maximum at magnitudes well above our optical limiting magnitudes. This turnover in the magnitude distribution of faint radio sources was initially hinted in the Leiden-Barkeley Deep Survey (LBDS) radio sample (Windhorst et al. 1984) and more recently confirmed in the LBDS Hercules subsample (Waddington et al. 2000) and in the identification of the radio sources in the Hubble Deep Field region (Richards et al. 1999).

In Fig. 4 we show the $20 \mathrm{~cm}$ radio flux versus the $I_{A B}$ band magnitude for all the radio sources with an optical identification. Superimposed are the lines corresponding to constant values for the observed radio-to-optical ratios $R$ defined as $R=S \times$ $10^{0.4(m a g-12.5)}$, where $S$ and mag are the radio flux in mJy and the apparent magnitude of the optical counterparts respectively. In Fig. 5 we show the $I_{A B}$ and $(V-I)_{A B}$ distributions in different radio flux bins. The shaded histograms in the $I_{A B}$ magnitude distributions show the number of unidentified radio sources, arbitrarily plotted in four equal bins between $I_{A B}=25.0$ and $I_{A B}=27.0$.

In Table 4, for each radio flux bin, we report the mean, median and standard deviations for the $I_{A B}^{\text {med }}$ and $(V-I)_{A B}^{\text {med }}$ color distributions of all the identified radio sources, the number of radio sources with an optical identification, the total number of radio sources (excluding the sources outside the CCD area and the sources in the masked regions, see Sect. 3), the percentage of radio sources with a reliable optical identifications and the median $I_{A B}^{\text {med+unid }}$ magnitude calculated considering also the unidentified sources, all assumed fainter than $I_{A B}=25.0$.

Figure 4 shows an extremely large scatter between radio flux and magnitude, while the analysis of the optical properties in different radio flux bins (see Table 4) shows a mean (and median) optical magnitude that becomes fainter as fainter radio flux bins are considered, although this trend is not statistically significant due the large spread of the distributions (see Col. 4 (standard deviations) in Table 4)

However, while about $35 \%$ of the radio sources are optically unidentified in the first radio flux bin (see Fig. 5 and Table 4), the percentage of unidentified sources decreases to about $25 \%$ in the faintest two radio bins. Because of this decrease of unidentified sources, although within the Poisson errors, the median $I_{A B}$ magnitude for the total sample of radio sources, i.e. including also the unidentified ones, is actually brighter of $\sim 0.6 \mathrm{mag}$ in the faintest radio bin than in the bin with higher radio flux (see last column in Table 4). This result shows that the faintest radio sources are not in general the faintest sources at optical wavelengths and would suggest that most of the faintest radio sources are likely to be associated to relatively low redshift star forming objects with a low radio luminosity, rather than radio-powerful, AGN type objects at high redshift. Their median photometric redshift $z_{\text {phot }}$ (see next section for more details on $z_{\text {phot }}$ ) is 0.67 , with $\sim 90 \%$ of the sources in the photometric redshift range $0.1 \leq z_{\text {phot }} \leq 1.5$. 

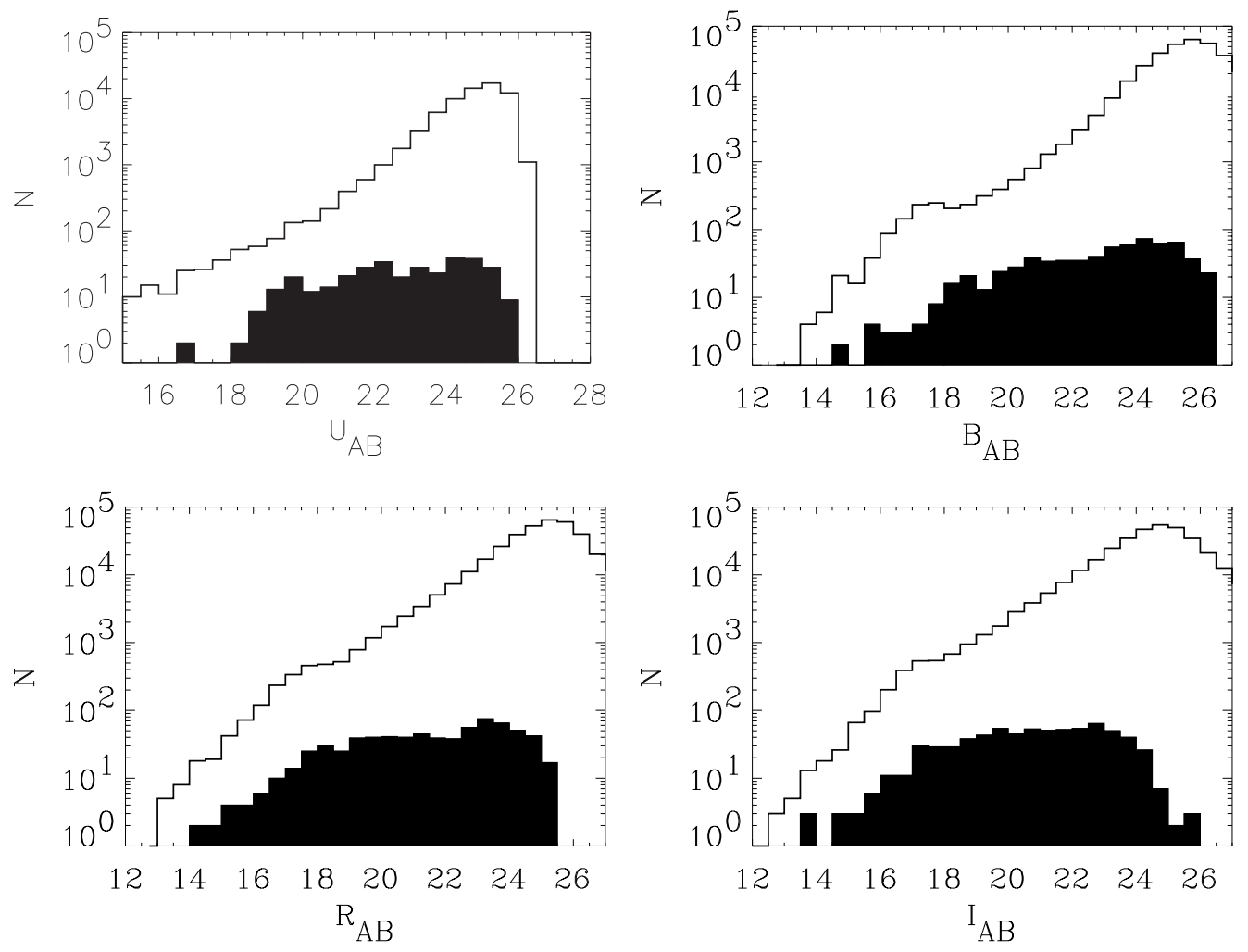

Fig. 3. Magnitude distributions in the $U_{A B}, B_{A B}, R_{A B}$, and $I_{A B}$ band for the optical counterparts of the radio sources (filled histograms) and for the whole optical sample (empty histograms).

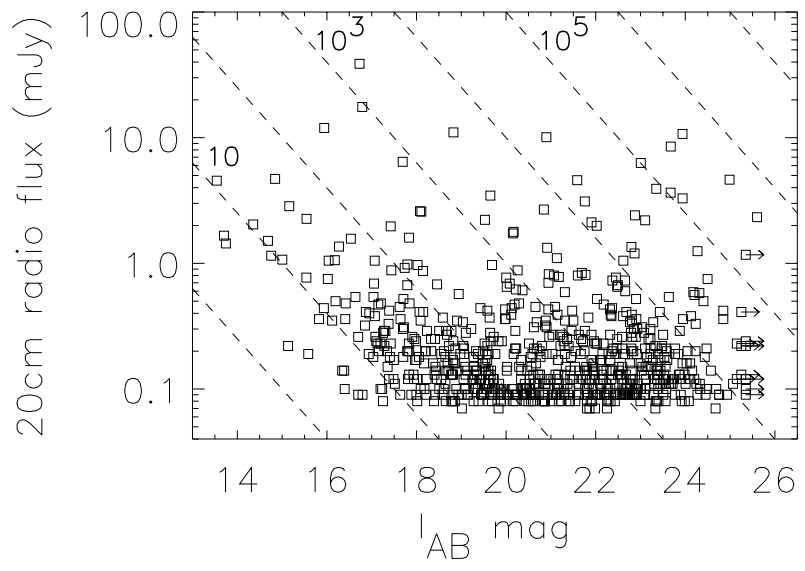

Fig. 4. The $I_{A B}$ band magnitude versus $20 \mathrm{~cm}$ radio flux for all the radio sources with a reliable optical identification. The lines represent different radio to optical ratio $R$, corresponding to $R=1,10,10^{2}, 10^{3}$, $10^{4}, 10^{5}, 10^{6}, 10^{7}$.

This result is consistent with the expectations from previous work in the optical identification of the radio sources. Many authors (Windhorst et al. 1995; Richards et al. 1998, 1999; Roche et al. 2002) have shown, in fact, that the majority of the optical identifications of the $\mu \mathrm{Jy}$ radio sources are with luminous $\left(L>L_{*}\right)$ galaxies at relatively modest redshifts $(0 \leq z \leq 1)$, many of which with evidence for recent star formation.

Finally, the analysis of the optical color $(V-I)_{A B}$ in different radio flux bins (see Fig. 5 and Table 4) shows that there is a small reddening of the median color as fainter radio fluxes are considered.

\subsection{Unidentified radio sources}

As described in Sect. 3, we have 255 radio sources (26\% of the radio sample) without optical counterpart in our optical and near infrared images. The presence of a significant fraction ( $25-30 \%)$ of radio sources with an optical magnitude fainter than $\sim 25$ was already noted by several authors in radio surveys of similar depth. In the identification of the microjansky radio sources in the HDF, only 84 (out of 111, 76\%) sources have been identified to $I=25 \mathrm{mag}$, with the bulk of the sample identified with relatively bright $(I \leq 22)$ galaxies (Richards et al. 1999), while in the Phoenix Deep Survey (PDS) about $79 \%$ of the sources (659 out of 839) have been identified using optical $U B V R I$ images to $R \sim 24.5$ (Sullivan et al. 2004).

The 255 unidentified VIMOS radio sources have a median radio flux of $0.15 \mathrm{mJy}$, equal to that of identified sources (see also Fig. 5 and Table 4 for their radio flux distribution). Given the very faint optical counterpart, these unidentified radio sources might contain a significant fraction of obscured and/or high redshift galaxies. Deep surveys performed at wavebands free from dust absorption (i.e. far-infrared and X-ray) may provide the most powerful tool to identify these objects. The VIRMOS VVDS-02h field is a selected target for farinfrared and X-ray observations with the Spitzer and XMM satellites in the framework of the Spitzer Legacy Programme 

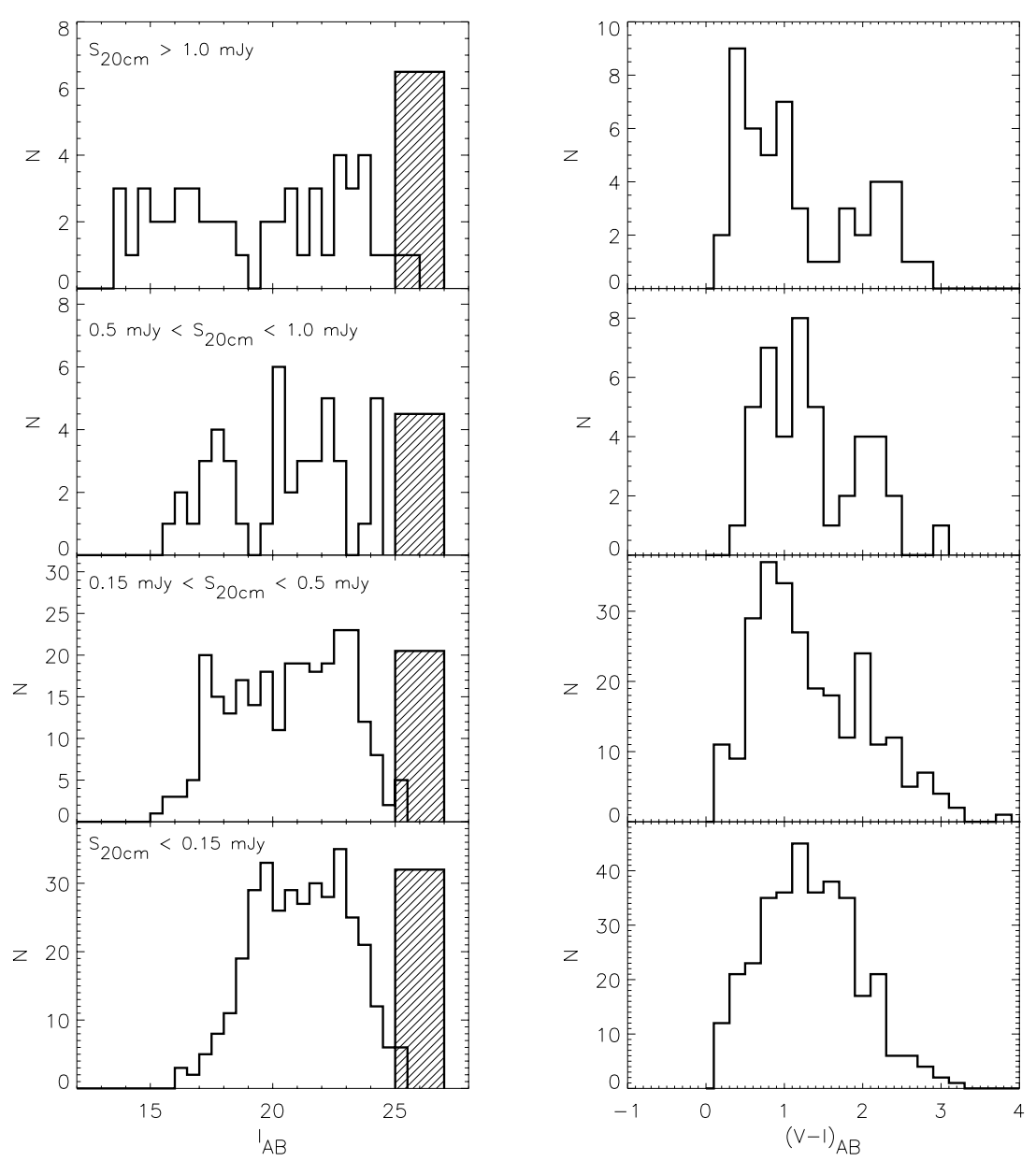

Fig. 5. The $I_{A B}$ magnitude (left) and optical color $(V-I)_{A B}$ (right) distributions in different radio flux bins for all the radio sources with a reliable optical identification. The shaded histograms in the left panels represent the unidentified radio sources.

Table 4. The $I_{A B}$ magnitude (mean, median and standard deviations) and $(V-I)_{A B}$ color (mean, median and standard deviations) in four different radio flux bins.

\begin{tabular}{|c|c|c|c|c|c|c|c|c|c|c|}
\hline Radio flux & mean & $\begin{array}{c}I_{A B} \\
\text { median }\end{array}$ & $\sigma$ & mean & $\begin{array}{c}(V-I)_{A B} \\
\text { median }\end{array}$ & $\sigma$ & $N_{\text {radio ID }}^{1}$ & $N_{\text {radio }}^{2}$ & $\%$ ID & $I_{A B}^{\mathrm{med}+\text { unid } 3}$ \\
\hline$S_{20 \mathrm{~cm}} \geq 1.00 \mathrm{mJy}$ & 19.32 & 19.65 & 3.55 & 1.15 & 0.97 & 0.94 & 51 & 77 & $66 \%$ & 22.9 \\
\hline $0.50 \leq S_{20 \mathrm{~cm}}<1.00 \mathrm{mJy}$ & 20.38 & 20.95 & 2.56 & 1.25 & 1.22 & 0.62 & 44 & 62 & $71 \%$ & 22.4 \\
\hline $0.15 \leq S_{20 \mathrm{~cm}}<0.50 \mathrm{mJy}$ & 20.59 & 20.89 & 2.31 & 1.29 & 1.18 & 0.76 & 268 & 350 & $77 \%$ & 22.0 \\
\hline$S_{20 \mathrm{~cm}}<0.15 \mathrm{mJy}$ & 21.14 & 21.18 & 1.90 & 1.25 & 1.26 & 0.71 & 355 & 483 & $74 \%$ & 22.3 \\
\hline
\end{tabular}

${ }^{1}$ Number of radio sources with a reliable optical counterpart.

2 Total number of radio sources with good CCD data.

${ }^{3}$ Median value obtained assuming all the unidentified radio sources with $I_{A B}>25.0$.

SWIRE (Lonsdale et al. 2003) and the XMM Large Scale Structure Survey (Pierre et al. 2004) respectively.

\subsection{Photometric redshifts}

The optical data in the $B_{A B}, V_{A B} R_{A B}$ and $I_{A B}$ bands, plus $U_{A B}$, $J_{A B}$ and $K_{A B}$ data (when available) allow us to estimate photometric redshifts for all the 718 radio sources with an optical counterpart. A detailed description and discussion of the method used to estimate the photometric redshift in the VIMOS survey is reported in Bolzonella et al. in preparation.
Photometric redshifts have been computed with two codes developed by the authors: Hyperz, by Bolzonella et al. (2000) ${ }^{3}$, using the Bruzual \& Charlot (2003) library, and the code Le Phare by Arnouts \& Ilbert ${ }^{4}$ using 72 CWW (Coleman et al. 1980) extended templates. Both of them have been modified allowing a training of photometric redshifts using the spectroscopic data.

The first step of the method consists in training the photometry: observations are affected by possible uncertainties on

\footnotetext{
${ }^{3}$ http://webast.ast.obs-mip.fr/hyperz/

${ }^{4}$ http://www. lam.oamp.fr/arnouts/LE_PHARE.html
} 


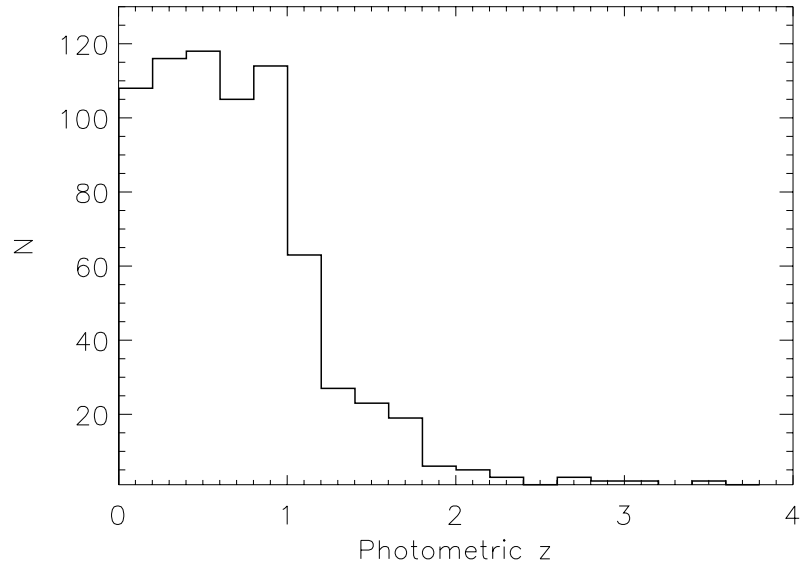

Fig. 6. The photometric redshift histogram for the 718 radio sources with a reliable optical counterpart.

photometric calibration and zeropoints. At the same time, the template SEDs may be not fully representative of the observed galaxy population and it may be difficult to reproduce precisely the response functions of filters. Therefore we calibrated photometry and templates with the spectroscopic sample in order to obtain a good agreement between the observed colourredshift relation and the one derived from templates.

Moreover, to avoid spurious solutions at high redshift, frequently found when the $U$ magnitude is not available, we imposed a prior in the redshift distribution. To this aim we used the formalism described in Benítez (2000), without considering the mix of spectrophotometric types. We applied a prior roughly reproducing the $N(z)$ of the spectroscopic sample, but with a non null probability of being at high redshift. By imposing this prior we do not significantly affect the redshift distribution and we considerably improve the agreement between photometric and spectroscopic redshifts, in particular when only the optical magnitudes are available. The values of photometric redshifts used in this paper have been obtained with the second of the two codes, Le Phare, although the two codes produced very similar results.

So far, only a small fraction $(54 / 718)$ of the optical counterparts of the radio sources has a spectroscopically measured redshift. For these sources the spectroscopic and photometric redshifts are in good agreement $(90 \%$ of the objects have $\left.\Delta z /\left(1+z_{\text {spec }}\right)<0.07\right)$. The analysis of the sub-sample of spectroscopically identified radio-sources is in progress and will be presented elsewere.

Figure 6 shows the photometric redshift histogram for all the radio sources with a reliable optical counterpart. About $80 \%$ of the sources are estimated to be at $z \leq 1$, with a small high redshift tail extending up to $z \sim 3$, although among the 157 radio sources with $z_{\text {phot }}>1.0$ we have the $J$ and $K$ bands data (and then a more reliable $z_{\text {phot }}$ ) for only 11 sources. This redshift distribution is in good agreement with that obtained by Sullivan et al. (2004) in their analysis of the optical and near infrared counterparts of the radio sources in the Phoenix Deep Survey.

\subsection{Color-color diagrams and comparison with the whole optical data set}

In this section we compare the optical colors properties of the radio sources with those of the whole optical data set. A detailed discussion of the color properties of the whole optical data set with a comparison to those from other deep surveys is reported in McCracken et al. (2003).

In Fig. 7 we show the $(B-V)_{A B}$ and $(V-I)_{A B}$ colors for the whole data set (small dots) and for the optical counterparts of the radio sources (open squares). The sources classified as point-like in the optical band $\left(I_{A B}<21.5\right)$ are plotted in the top-left panel, while the sources classified as extended are plotted in the other three panels in three different magnitude ranges $\left(18<I_{A B}<20,20<I_{A B}<22\right.$ and $22<I_{A B}<24$, respectively). Solid, dashed and dashed dot-dot-dot lines show the path in redshift of early-type, Sab and Sbc galaxies. The evolutionary tracks were computed using the "2000" revision of the GISSEL libraries (Bruzual \& Charlot, 1993). The median value of $(B-V)_{A B},(V-I)_{A B}, z_{\text {phot }}$ and $I_{A B}$ for the sources classified as extended are reported in Table 5, where the same values are given also for the whole optical sample in the same magnitude ranges. The errors on the median values reported in Table 5 have been calculated using $1.2533 \sigma / \sqrt{N}$, where $\sigma$ is the standard deviation of the distribution and $N$ is the number of objects (Akin \& Colton 1970).

Moreover, for each data set $\left((B-V)_{A B},(V-I)_{A B}, z_{\text {phot }}\right.$ and $\left.I_{A B}\right)$ in each magnitude bin, the Kolmogorov-Smirnov test (KS) has been used to test the hypothesis that the two distributions (that from the whole optical sample and that from the radio sources) are drawn from the same distribution. The results of the KS test are reported in Table 5 (last row in each magnitude bin). In particular, for each KS test performed, we report the significance level of the KS statistic. Small values show that the two distributions are significantly different.

The color-color plot for point-like sources (top-left panel of Fig. 7) shows that, as expected, the majority of the radio sources with a point-like optical counterpart have different colors with respect to the bulk of the whole optical data set. In fact, while the majority of the point-like sources are expected to be stars, those associated with radio sources are, with very high probability, active galactic nuclei (AGN) or actively star forming compact galaxies.

The top-right panel of Fig. 7 shows the bright magnitude slice at $18<I_{A B}<20$ for extended sources. As shown in the Figure and in Table 5 the color properties of the radio sources in this bright magnitude slice are not significantly different from those of the whole optical data set. The bulk of the optical and radio sources are consistent with model tracks of a galaxy population around $z \sim 0.2-0.3$ but the median photometric redshift of the radio sources is higher than that of the whole optical sample (see Table 5).

The lower-left panel of Fig. 7 shows the intermediate magnitude slice at $20<I_{A B}<22$. As already noted by McCracken et al. (2003), the whole galaxy population (predominantly latetype galaxies) occupies two distinct loci, and in this color-color space there is a reasonably well defined separation between high $(z>0.4)$ and low $(z<0.4)$ redshift galaxies. However, as 

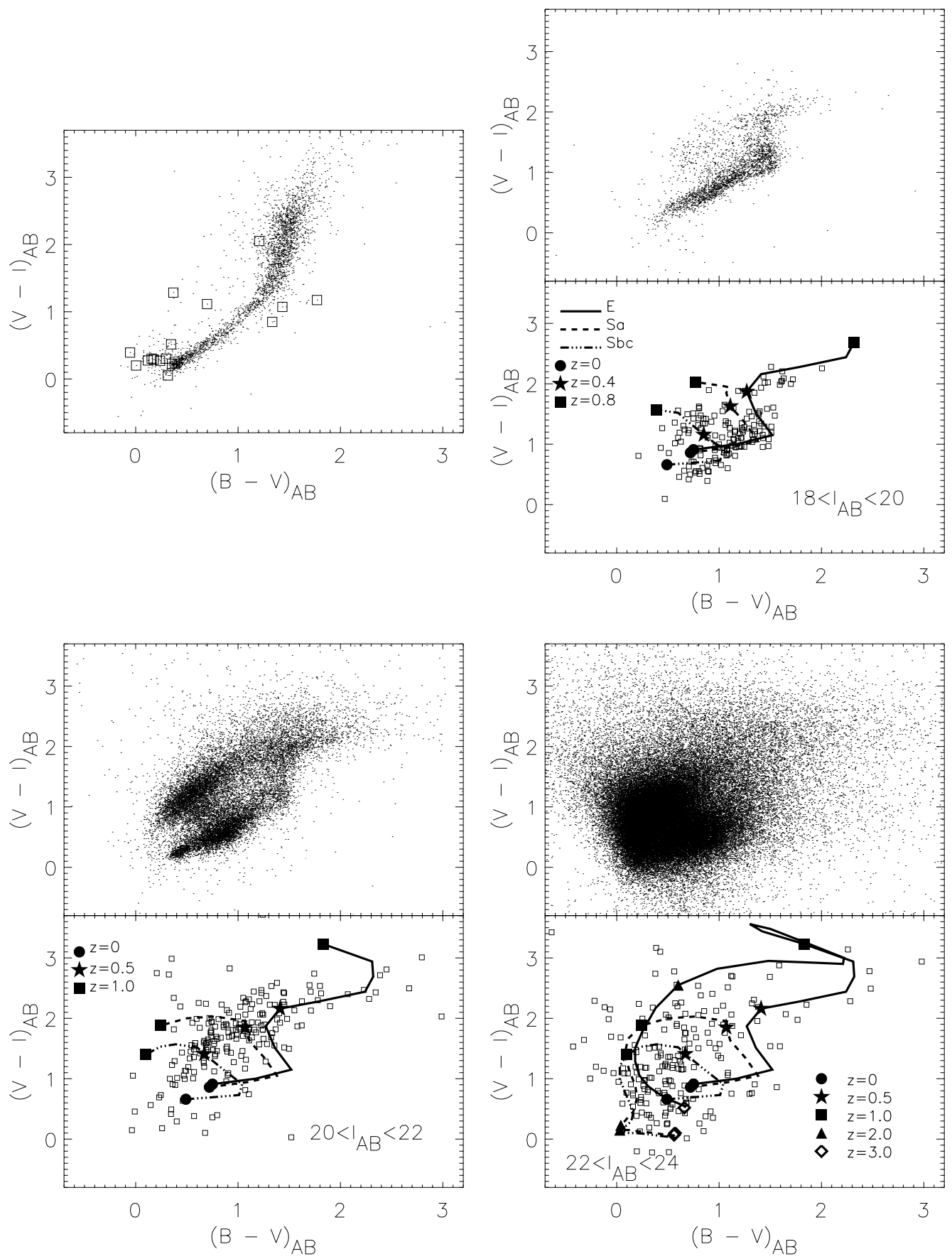

Fig. 7. $(B-V)_{A B}$ and $(V-I)_{A B}$ colors for the whole data set (small dots) and for the optical counterparts of the radio sources (open squares). The sources classified as point-like in the optical band $\left(I_{A B}<21.5\right)$ are plotted in the top-left panel, while the sources classified as extended are plotted in the other three panels in three different magnitude ranges. Solid, dashed and dashed dot-dot-dot lines show the path in redshift of early-type, Sa and Sbc galaxies. The evolutionary tracks were computed using the "2000" revision of the GISSEL libraries (Bruzual \& Charlot 1993). The model tracks span the redshift range $0.0<z<0.8$ for the magnitude slice $18<I_{A B}<20,0.0<z<1.0$ for the magnitude slice $20<I_{A B}<22$ and $0.0<z<3.0$ for the magnitude slice $22<I_{A B}<24$.

clearly shown in the figure, the radio sources essentially populate only the locus of the high redshift $(z \sim 0.4-0.5)$ galaxies. This is confirmed by the photometric redshift analysis, with a median redshift of the whole data sample (0.56) significantly lower than the median redshift of the radio sample $(0.82$; see Table 5). The median $(V-I)_{A B}$ color of the radio sources $\left((V-I)_{A B}^{\mathrm{med}}=1.77\right.$, see Table 5) is also significantly redder than that of the whole optical data set $\left((V-I)_{A B}^{\mathrm{med}}=1.16\right)$. The
KS test shows that the $(V-I)_{A B}, z_{\text {phot }}$ and $I_{A B}$ distributions of the whole optical sample are very significantly different from that of the radio sources (see Table 5).

Finally, in the fainter magnitude slice $\left(22<I_{A B}<24\right.$, lower-right panel of Fig. 7) the colors are bluer than in the brighter magnitude slice both for the radio sources and the whole optical data set. Even if less evident from the figure, also in this magnitude slice there is a statistically significant 
Table 5. The median values for $(B-V)_{A B},(V-I)_{A B}$, photometric redshift $z_{\text {phot }}$ and $I_{A B}$ magnitude in three different magnitude slices for the whole optical data set and for the optical counterparts of the radio sources. The last column reports the number of sources in the magnitude bin.

\begin{tabular}{|c|c|c|c|c|c|}
\hline Sample & $(B-V)_{A B}$ & $(V-I)_{A B}$ & $z_{\text {phot }}$ & $\overline{I_{A B}^{\mathrm{med}}}$ & $\overline{N N}$ \\
\hline \multicolumn{6}{|c|}{ Magnitude range $18<I_{A B}<20$} \\
\hline Optical sample & $1.10 \pm 0.01$ & $1.06 \pm 0.03$ & $0.31 \pm 0.01$ & $19.31 \pm 0.01$ & 3056 \\
\hline Radio sources & $1.08 \pm 0.03$ & $1.17 \pm 0.04$ & $0.39 \pm 0.02$ & $19.22 \pm 0.05$ & 153 \\
\hline $\mathrm{KS}$ test significance & 0.13 & 0.005 & 0.04 & 0.04 & \\
\hline \multicolumn{6}{|c|}{ Magnitude range $20<I_{A B}<22$} \\
\hline Optical data set & $0.82 \pm 0.01$ & $1.16 \pm 0.01$ & $0.56 \pm 0.01$ & $21.32 \pm 0.01$ & 17962 \\
\hline All radio sources & $0.93 \pm 0.03$ & $1.77 \pm 0.06$ & $0.82 \pm 0.04$ & $21.03 \pm 0.05$ & 190 \\
\hline $\mathrm{KS}$ test significance & 0.003 & $<1 \times 10^{-8}$ & $<1 \times 10^{-8}$ & $3.6 \times 10^{-6}$ & \\
\hline \multicolumn{6}{|c|}{ Magnitude range $22<I_{A B}<24$} \\
\hline Optical sample & $0.46 \pm 0.01$ & $0.88 \pm 0.01$ & $0.80 \pm 0.01$ & $23.33 \pm 0.01$ & 82944 \\
\hline Radio sources & $0.53 \pm 0.05$ & $1.22 \pm 0.07$ & $0.96 \pm 0.05$ & $22.87 \pm 0.05$ & 206 \\
\hline $\mathrm{KS}$ test significance & 0.03 & $<1 \times 10^{-8}$ & $<1 \times 10^{-8}$ & $<1 \times 10^{-8}$ & \\
\hline
\end{tabular}

difference between the color, photometric redshift and magnitude distributions of the radio sources and the radio quiet galaxies (see results of KS test in Table 5).

The fact that, in each magnitude bin, the median photometric redshift of the radio sample is higher than that of the whole optical sample shows that radio detection is preferentially selecting galaxies with higher intrinsic optical luminosity. This conclusion is strengthened by the fact that, because of the different magnitude distributions (see Fig. 3), the median magnitude $I_{A B}^{\mathrm{med}}$ of the radio sample is brighter than that of the optical sample in each magnitude bin (see Col. 5 in Table 5).

\subsection{Color-redshift diagram for the radio sample}

In Fig. 8 we show the optical color $(B-I)_{A B}$ versus the photometric redshift for all the radio sources with an optical counterpart, together with predicted loci for early type galaxies (solid line), Sa galaxies (dashed line) and Sbc galaxies (dashed dotdot-dot line). Sources above the Sa track are expected to be mainly early type galaxies and most of them (222/263) have $z_{\text {phot }} \geq 0.5$, while the sources below the Sa track are expected to be mainly late-type, star-forming galaxies and they more uniformly occupy the entire redshift range, including the low redshift one.

In order to study, at least statistically, the properties of the two classes of objects, we have adopted in the following the Sa track as separation between early and late type galaxies. We are fully aware that such a classification is only a first approximation, which does not work on an object-by-object basis, and can be significantly uncertain especially at redshift greater than $\sim 1$. In fact, above $z \sim 1$ the photometric redshifts are more uncertain. This, coupled with the rapidly decreasing expected $(B-I)_{A B}$ color of the Sa track up to $z \sim 2$, makes the separation between early and late type galaxies only indicative in this redshift range. A somewhat similar separation has been already

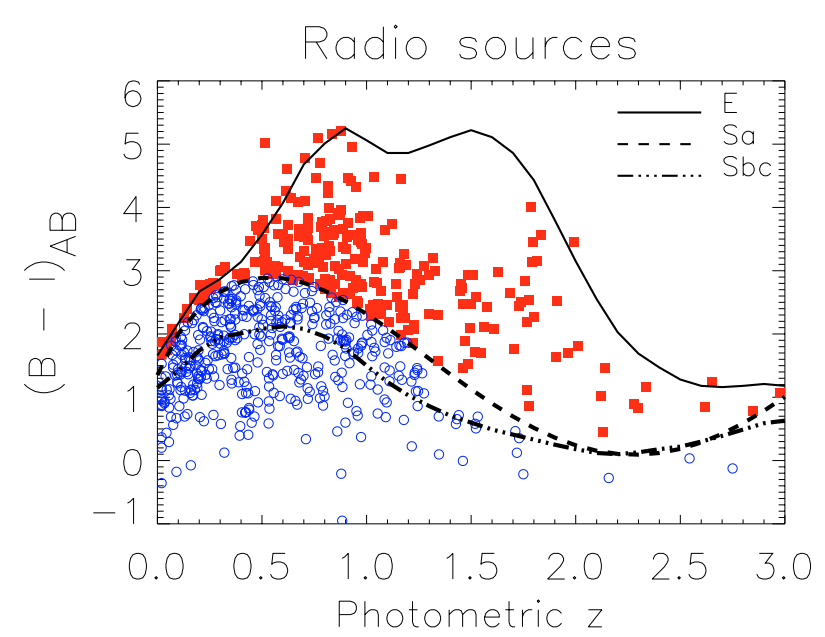

Fig. 8. The $(B-I)_{A B}$ color versus the photometric redshift for the radio sources with a reliable optical counterpart. Sources above the Sa track (expected to be mainly early type galaxies at $z \geq 0.5$ ) are plotted with filled squares, while the sources below the Sa track (expected to be mainly late-type, star-forming galaxies at all redshift) are plotted with empty circles (see text for more details).

used by Ciliegi et al. (2003) in their analysis of the $6 \mathrm{~cm}$ radio sources in the Lockman Hole. They used the color $V-K=5.2$ to separate the high redshift $(z \geq 0.5)$ early-type galaxies from the late-type, star forming galaxies at all redshifts. Due to the lack of the $K$-band covering for the whole VIMOS radio sample, we cannot use the $V-K$ color to separate the two classes. However, as clearly shown in their Fig. 13, the two methods are largely equivalents: only two sources (out of 63 objects) have a $V-K$ color consistent with early-type galaxies $(V-K>5.2)$ but lie well below the Sa track.

We then used these two radio sub-samples selected on the basis of the Sa track to search for possible differences in the radio-to-optical ratio $R$ (defined as in Sect. 4.1) between early 


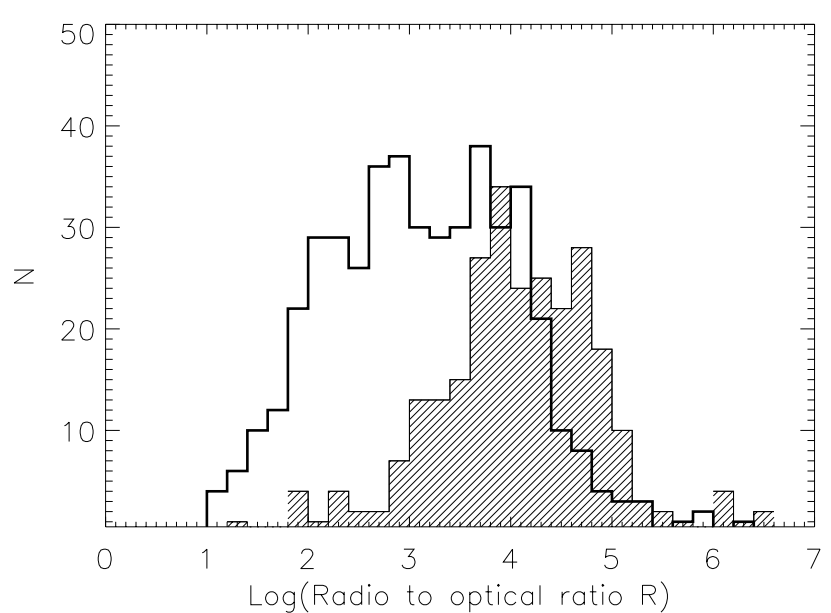

Fig. 9. Distribution of the radio-to-optical ratio $R$ for the sample of early type galaxies (shaded histogram) and for the sample of late type galaxies (empty histogram).

and late type galaxies. Previous works on the optical identifications of radio sources (Kron et al. 1985; Gruppioni et al. 1999; Richards et al. 1999; Geogakakis et al. 1999; Prandoni et al. 2001; Ciliegi et al. 2003) have suggested, in fact, that the majority of the sources with low radio-to-optical ratio are associated with star forming galaxies, characterized by moderately weak intrinsic radio power. Viceversa, early type galaxies, in which radio luminosity is likely connected to nuclear activity, cover a much larger range in radio power, and hence in $R$, becoming the dominant population at high $R$. In Fig. 9 we present the distribution of the radio-to-optical ratio $R$ for the two samples of early type galaxies (shaded histogram) and the sample of late type galaxies (empty histogram). The two distribution are very significantly different (at more than $7 \sigma$ level, on the basis of KS test): late-type, star forming galaxies are the dominant population at low radio-to-optical ratio $R$ (median $\log R=3.08 \pm 0.05$, using the $B_{A B}$ magnitude to calculate $R$ ), while the majority of the galaxies in the region of high $R$ are early-type galaxies (median $\log R=4.06 \pm 0.06$ ). In the high $R$ tail we still have a component of objects classified as late type galaxies (about one third of the objects with $\log R \geq 4.6$ ). A better understanding of the physical nature of these objects, which may include also star forming galaxies in which the high $R$ value is due to significant dust obscuration, requires spectroscopic data.

Finally, we estimated the relative fraction of the two populations (early and late type galaxies) in different radio flux bins. We used the four flux bins defined in Table 4. Assuming, consistently with Fig. 9, that about $2 / 3$ of the unidentified radio sources (which have, by definition, high $R$ values) are earlytype galaxies, we find that while in the first three radio flux bins the fraction of early type galaxies is approximately constant at $\sim 40 \%$, in the fainter radio flux bin this fraction decreases to $\sim 30 \%$. Therefore, although this result is still highly qualitative and based on very simple assumptions, we confirm that in the fainter radio flux limit bin our radio sample is likely to be dominated by late-type star forming galaxies as already suggested by the analysis of the optical magnitude distribution in different radio flux intervals (see Sect. 4.1).

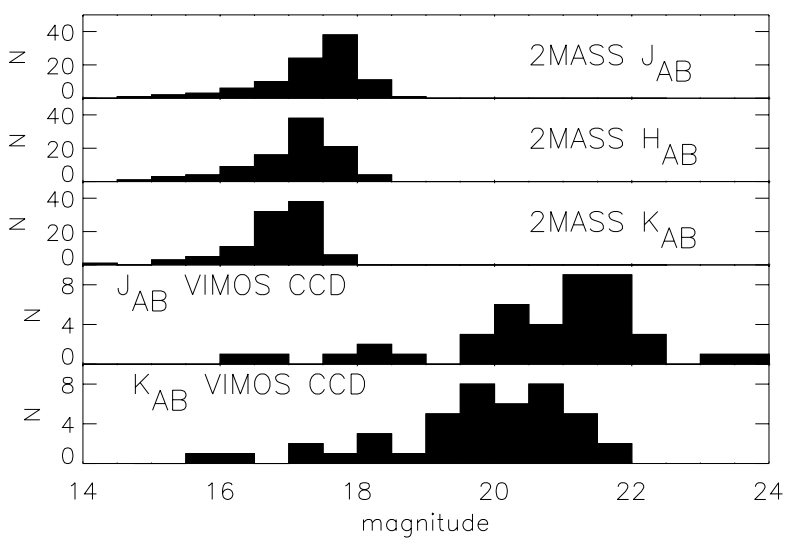

Fig. 10. The magnitude distributions in the three 2MASS near infrared bands for the 105 radio sources with a reliable conunterpart in the 2MASS catalogue and the magnitude distributions in the $J_{A B}$ and $K_{A B}$ bands for the near infrared counterparts of the 43 radio sources identified in the VIMOS near infrared survey (bottom panels). The 2MASS $J, H$, and $K$ magnitudes have been converted to the $A B$ magnitude system using the relations reported in Sect. 3.4.

\subsection{Near infrared magnitude properties}

In Fig. 10 we report the magnitude distributions in the three 2MASS bands for the 105 sources with an identification in the 2MASS catalogue and the $J_{A B}$ and $K_{A B}$ distributions for the 43 radio sources identified with the VIMOS near infrared survey. Moreover Fig. 11 shows the $(I-K)_{A B}$ color as function of the $I_{A B}$ magnitude. In considering these figures, it must be remembered how incomplete these data are: only 65 of the radio sources are within the area covered by the VVDS $J$ and $K$ bands survey, while the radio flux distribution of the radio sources with a 2 MASS counterpart is strongly biased towards high radio flux due to the bright limit of the 2MASS survey. Figure 11 clearly shows that there is a monotonic trend for the optically fainter radio sources to have redder opticalinfrared colors, and their color reaches $(I-K)_{A B} \geq 2$ at $I_{A B} \sim 23 \mathrm{mag}$. Similar results have been obtained for different radio surveys (Richards et al. 1999; Waddington et al. 2000; Ciliegi et al. 2003). From Fig. 11 it is also interesting to note that only $7 \%(3 / 43)$ of the radio sources are very red sources with $(I-K)_{A B}>3.0$. An even smaller percentage of EROS has been recently obtained by Sullivan et al. (2004) during the optical and near infrared identification of the radio sources in the Phoenix Deep Survey. None of the 91 radio sources detected in the $K$ band (over a total of 111 radio sources) has an $(I-K)_{A B}$ color greater than 3.0 and only one source has an $(I-K)_{A B}>2.5$. The fact that EROs sources are only a few percent of the total number of counterparts in radio surveys with a flux limit around $\sim 100 \mu \mathrm{Jy}$ is not surprising. Deep radio observations of optically/near-infrared selected EROS have shown that the EROs population is not a class of strong radio sources. For example, Smail et al. 2002, using very deep $1.4 \mathrm{GHz}$ radio data $(3.5 \mu \mathrm{Jy}$ at $1 \sigma)$ found only 3 EROs (over a total of 68) with a radio flux greater than $85 \mu \mathrm{Jy}$, while Cimatti et al. 2003 studying a sample of 47 EROs, detected only one 


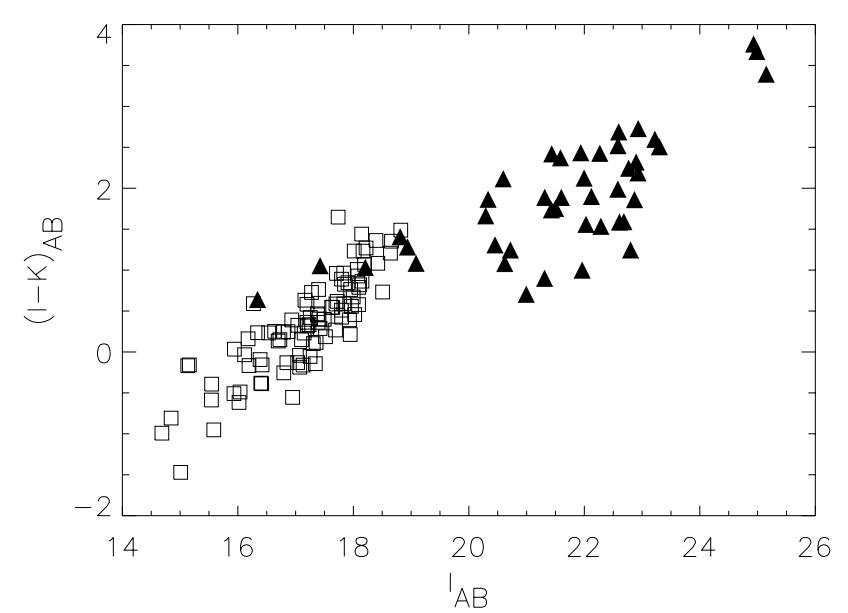

Fig. 11. The $(I-K)_{A B}$ color as function of the $I_{A B}$ magnitude. Open squares are sources from the 2MASS data while filled triangles are sources from the VIMOS $K$ band survey.

object at $1.4 \mathrm{GHz}$ with a radio flux of $\sim 106 \mu \mathrm{Jy}$. However, the correlation between color and magnitude shown in Fig. 11 suggests that the fraction of EROs is likely to be higher among the unidentified radio sources. This can be tested with deeper optical and infrared data.

\section{Summary and conclusion}

In this paper we have presented the optical and near-infrared identifications of the 1054 radio sources detected in the $20 \mathrm{~cm}$ deep radio survey obtained with the VLA in the VIMOS VLT Deep Survey VVDS-02h deep field (VVDS). Almost the whole square degree of the VVDS-VLA field has been observed in the $B, V, R$ and $I$ band down to a limiting magnitude of $B_{A B} \sim 26.5, V_{A B} \sim 26.2, R_{A B} \sim 25.9$ and $I_{A B} \sim 25.0$ (McCracken et al. 2003). Moreover, $\sim 0.71 \mathrm{deg}^{2}$ have been observed in the $U$ band down to $U_{A B} \sim 25.4$ (Radovich et al. 2004) and $\sim 165 \operatorname{arcmin}^{2}$ have been observed in the $J$ and $K$ bands down to $J_{A B} \sim 24.2$ and $K_{A B} \sim 23.9$ (Iovino et al. 2005). Using the Likelihood Ratio technique we optically identified 718 radio sources ( $\sim 74 \%$ of the whole sample). Sixtyfive radio sources lie within the $K$ band area and we found a reliable counterpart for 43 of them. Among the 255 unidentified radio sources, 17 are empty fields (i.e. they have no optical source within 5 arcsec from their position), while the other 238 sources have at least one optical source within 5 arcsec but all with $L R<L R_{\text {th }}$.

The color properties of the optical counterparts of the radio sources have been analysed using the $(B-V)_{A B}$ and $(V-I)_{A B}$ colors. The optical counterparts of the radio sources classified as extended have been analysed in three different magnitude slices. While in the brightest magnitude range $(18<$ $\left.I_{A B}<20\right)$ the optical color properties of the radio sources are not significantly different from those of the whole optical sample, at fainter magnitude the median $(V-I)_{A B}$ color of the radio sources is redder than the median color of the whole optical sample, suggesting a higher redshift for the radio sources. This is also supported by the photometric redshift analysis which shows that, in each magnitude bin, the radio sample has a higher median photometric redshift than the whole optical sample. This suggests that radio detection is preferentially selecting galaxies with higher intrinsic optical luminosity.

Using the $(B-I)_{A B}$ color and the photometric redshift for all the radio sources with a reliable optical counterpart, we have tentatively divided the radio sample in two sub-samples: the sources above the Sa galaxies track have been considered as early type galaxies, while the sources below the same track have been considered as late type galaxies. The analysis of the radio-to-optical ratio $R$ of the two sub-samples confirms with high statistical significance the results already obtained by other authors : late-type, star forming galaxies are the dominant population at low $R$, while in the region of high $R$ the majority of these objects are early-type galaxies.

From the analysis of the optical properties of the radio sources in different radio flux bins, we found that while about $35 \%$ of the radio sources are optically unidentified in the first radio flux bin, the percentage of unidentified sources decreases to about $25 \%$ in the faintest two bins $(S<0.5 \mathrm{mJy})$. The median $I_{A B}$ magnitude for the total sample of radio sources, i.e. including also the unidentified ones, is brighter in the faintest two radio bins than in the bin with higher radio flux. This result shows that the faintest radio sources are not in general the faintest sources at optical wavelengths and would suggest that most of the faintest radio sources are likely to be associated to relatively lower radio luminosity objects at relatively modest redshift, rather than radio-powerful, AGN type objects at high redshift. Using the above classification in early-type and latetype galaxies we found that the majority of the radio sources below $\sim 0.15 \mathrm{mJy}$ are indeed late-type star forming galaxies in the photometric redshift range $0.1 \leq z_{\text {phot }} \leq 1.5$. These results are in agreement with the results obtained by several authors: the majority of the optical identification of the $\mu \mathrm{Jy}$ radio sources are with luminous $\left(L>L_{*}\right)$ galaxies at modest redshifts $(0 \leq z \leq 1)$, many of which with evidence for recent star formation (Windhorst et al. 1995; Richards et al. 1998, 1999, Roche et al. 2002).

Finally, as already noted by other authors for different radio surveys, we found a monotonic trend for the optically fainter radio sources to be associated with redder galaxies. In the area covered by $K$ data 3 out of 43 radio sources with a likely $K$ band counterpart have very red $(I-K)_{A B}$ colors.

The analysis of the sub-sample of spectroscopically identified radio-sources is in progress and will be presented elsewhere.

Acknowledgements. This research has been developed within the framework of the VVDS consortium.

This work has been partially supported by the Italian Ministry (MIUR) grants COFIN2003 (num.2003020150).

This publication makes use of data products from the Two Micron All Sky Survey, which is a joint project of the University of Massachusetts and the Infrared Processing and Analysis Center/California Institute of Technology, funded by the National Aeronautics and Space Administration and the National Science Foundation. 


\section{References}

Akin, H., \& Colton, R. R. 1970, Statistical Methods, 5th edn. (New York: Barnes \& Nobles)

Benítez, N. 2000, ApJ, 536, 571

Benn, C. R., Rowan-Robinson, M., McMahon, R. G., Broadhurst, T. J., \& Lawrence, A. 1993, MNRAS, 263, 98

Bolzonella, M., Miralles, J. M., \& Pelló, R. 2000, A\&A, 363, 476

Bondi, M., Ciliegi, P., Zamorani, G., et al. 2003, A\&A, 403, 857

Bruzual, A. G., \& Charlot, S. 1993, ApJ, 405, 538

Bruzual, A. G., \& Charlot, S. 2003, MNRAS, 344, 1000

Ciliegi, P., Zamorani, G., Hasinger, G., et al. 2003, MNRAS, 398, 901

Cimatti, A., Daddi, E., Cassata, P., et al. 2003, A\&A, 412, L1

Coleman, G. D., Wu, C. C., \& Weedman, D. W. 1980, ApJS, 43, 393

de Ruiter, H. R., Willis, A. G., \& Arp, H. C. 1977, A\&AS, 28, 211

Georgakakis, A., Mobasher, B., Cram, L., et al. 1999, MNRAS, 306, 708

Gruppioni, C., Mignoli, M., \& Zamorani, G. 1999, MNRAS, 304, 199

Hammer, F., Crampton, D., Lilly, S. J., Le Fèvre, O., \& Kenet, T. 1995, MNRAS, 276, 1085

Kron, R. G., Koo, D. C., \& Windhorst, R. A. 1985, A\&A, 146, 38

Le Fèvre, O., Mellier, Y., McCracken, H. J., et al. 2004a, A\&A, 417, 839

Le Fèvre, O., Vettolani, G., Garilli, B., et al. 2004b, A\&A, 439, 845
Lonsdale, C. J., Smith, H. E., Rowan-Robinson, M., et al. 2003, PASP, 115,897

McCracken, H. J., Radovich, M. , Bertin, E., et al. 2003, A\&A, 410, 17

Pierre, M., Valtchanov, I., Altieri, B., et al. 2004, JCAP, 09, 11

Prandoni, I., Gregorini, L., Parma, P., et al. 2001, A\&A, 369, 787

Prestage, R. M., \& Peacock, J. A. 1983, MNRAS, 204, 355

Radovich, M., Arnaboldi, M., Ripepi, V., et al. 2004, A\&A, 417, 51

Richter, G. A. 1975, Astron. Nachr., 296, 65

Richards, E. A., Kellermann, K. I., Fomalont, E. B., Windhorst, R. A., \& Partridge, R. B. 1998, AJ, 116, 1039

Richards, E. A., Fomalont, E. B., Kellermann, R. A., et al. 1999, ApJ, 526, L73

Roche, N. D., Lowenthal, J. D., \& Koo, D. C. 2002, MNRAS, 330, 307

Smail, I., Owen, F. N., Morrison, G. E., Keel, W. C., Ivison, R. J., \& Ledlow, M. J. 2002, ApJ, 581, 844

Sullivan, M., Hopkins, A. M., Afonso, J., et al. 2004, ApJS, 155, 1

Sutherland, W., \& Saunders, W. 1992, MNRAS, 259, 413

Szalay, A. S., Connolly, A. J., \& Szokoly, G. P. 1999, AJ, 117, 68

Waddington, I., Windhorst, R. A., Dunlop, J. S., Koo, D. C., \& Peacock, J. A. 2000, MNRAS, 317, 801

Windhorst R. A., van Heerde G. M., \& Katgert, P. 1984, A\&AS, 58, 1 Windhorst, R. A., Fomalont, E. B., Kellermann, K. I., et al. 1995, Nature, 375, 471 